\title{
Three Steps, Two Enzymes, One Pot, but a Multitude of Nanocompartments: Combined Cycles of Kinetic Resolutions and Re-racemization with Incompatible Biocatalysts
}

\author{
Florian Golombek, Marco Haumann, Matthias S.G. Knoll, Andreas Paul Fröba, and Kathrin Castiglione*
}

Cite This: ACS Omega 2021, 6, 29192-29200

Read Online

ABSTRACT: Deracemizations are clearly preferable to kinetic resolutions in the production of chiral molecules from racemates, as they allow up to $100 \%$ chemical and optical yield. Here we present a new process route for multienzymatic deracemizations that is relevant for reaction systems with incompatible reaction conditions of the biocatalysts. This often applies to combinations of lipases used for stereoselective acylation and solventsensitive racemases. By encapsulating a model racemase in polymeric vesicles, it was protected from inactivation by the organic solvent up to phase proportions of $99 \%$. As high yields in the lipase reaction required either water proportions well below $1 \%$ or racemasedenaturating acyl donor concentrations, a one-pot reaction was implemented through the sequential use of lipase and racemase-containing nanocompartments. This strategy allowed us to perform two kinetic resolutions with intermittent re-racemization in one pot yielding $72 \%(0.72 \mathrm{mM}$ after $120 \mathrm{~h})$ of an enantiopure product.

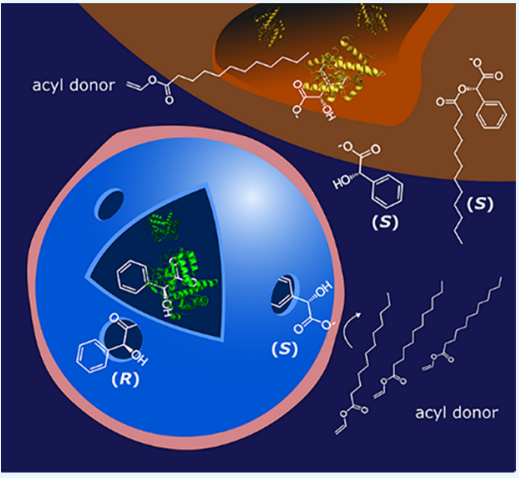

\section{INTRODUCTION}

Chiral compounds are important building blocks and primary targets in the manufacture of various chemicals, such as drugs, fragrances, agrochemicals, and food additives. ${ }^{1}$ A common method for the production of enantiopure substances is the kinetic resolution (KR) of racemates, where only one enantiomer is converted to a specific product by a stereoselective catalyst. However, since the theoretical yield cannot exceed a maximum of $50 \%$ in $\mathrm{KR}$, deracemization techniques that produce chiral molecules in up to $100 \%$ chemical and optical yield are much more attractive for industrial applications. $^{2,3}$ Dynamic kinetic resolution (DKR) is the most frequently applied method in this field. Here, the nonconverted substrate enantiomer is racemized in situ by a second catalyst during the $\mathrm{KR}$, affording the final product in quantitative yield.

The implementation of a DKR is often challenging since both involved catalysts have to work in the same environment. In situ racemization by acids, bases, or metal catalysts can lead to (bio)catalyst inactivation, which makes the use of a second enzyme for isomerization attractive. Although one could suspect that the intrinsic compatibility of the reaction conditions of two enzymes is better compared to chemical racemization, incompatibilities also occur here, especially if lipases are involved. ${ }^{4,5}$ Lipases are widely used for (D)KRs because they catalyze numerous reactions with excellent enantioselectivity, such as the esterification, transesterification, hydrolysis, or aminolysis of an alcohol, ester, or amine. ${ }^{2,4,6,7}$ The challenge of incompatibility is particularly high in reaction systems in which the lipase works in organic solvents since the enzymes employed for isomerizations are typically solventsensitive. ${ }^{4}$ In such cases, repeated KR with intermittent racemization represents an alternative process route for deracemization, which, however, is less powerful due to the necessary intermediate purifications. ${ }^{3}$

A well-known example of an enzyme system with incompatible reaction conditions is the resolution of $(R, S)$ mandelate by the lipase from Burkholderia cepacia (BCL) and the mandelate racemase (MR) from Pseudomonas putida. While the BCL needs organic solvents for interfacial activation $^{8}$ and very low water proportions to shift the equilibrium to the acylation reaction, the $M R$ is highly solvent-sensitive. ${ }^{9}$ Several attempts have been made in the past to overcome this challenge, such as the replacement of organic solvents by ionic liquids ${ }^{9}$ or the spatial separation of both enzymes using a hollow fiber membrane reactor. ${ }^{10}$ When using ionic liquids, MR was catalytically active up to a phase proportion of $90 \%$ but was inactivated by the acyl donor vinyl acetate, thus yielding no additional product compared to the classical KR. ${ }^{9}$ In contrast, the yield in the membrane reactor was $65 \%$, indicating the potential of spatial separation of the two enzymes but leaving significant room for improvement. ${ }^{10}$

Received: August 27, 2021

Accepted: October 4, 2021

Published: October 21, 2021 
Scheme 1. One-Pot Deracemization of $(R, S)$-Mandelate. ${ }^{a}$

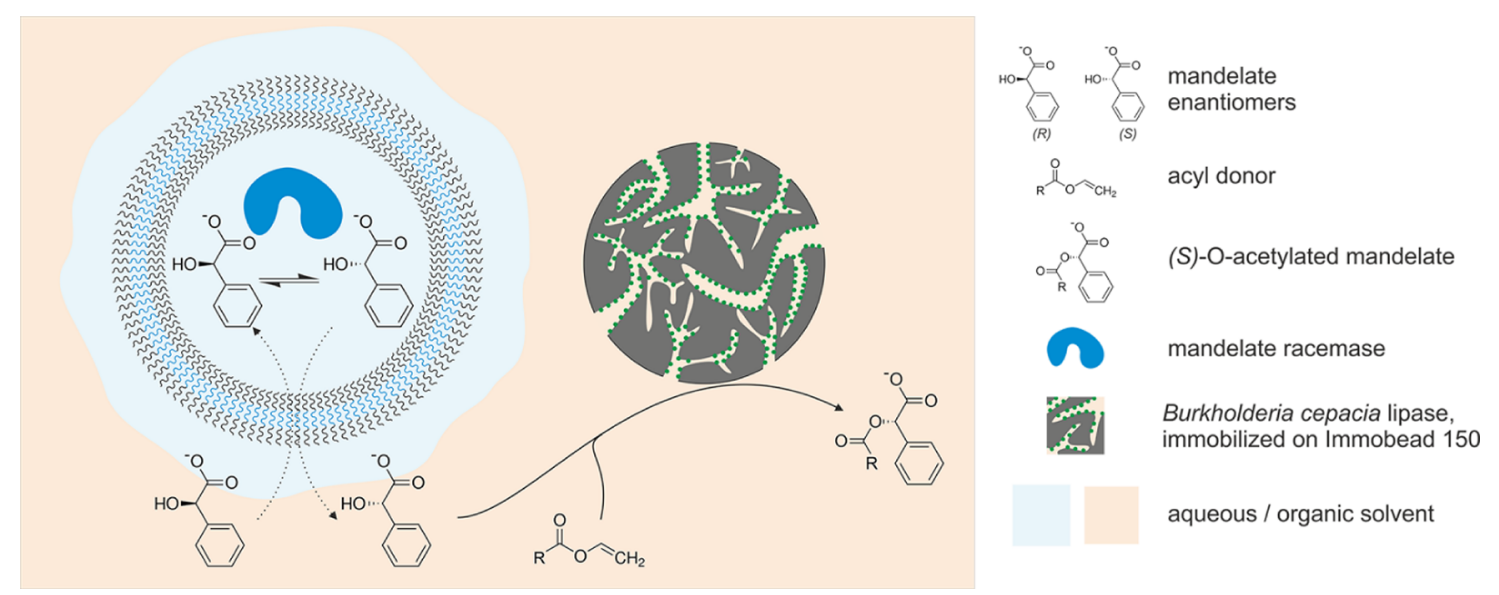

${ }^{a}$ While $(S)$-mandelate is converted via lipase-catalyzed $O$-acylation, the $(R)$-enantiomer is racemized in the aqueous phase. The solvent-sensitive racemase is encapsulated in polymeric vesicles, shielding the enzyme from the organic bulk phase but simultaneously enabling the transport of the mandelate through the membrane.

a)

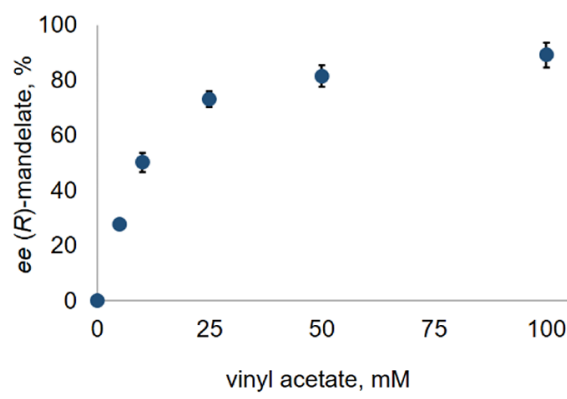

c)

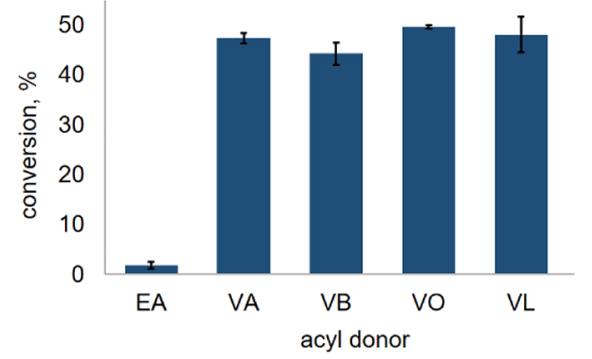

b)

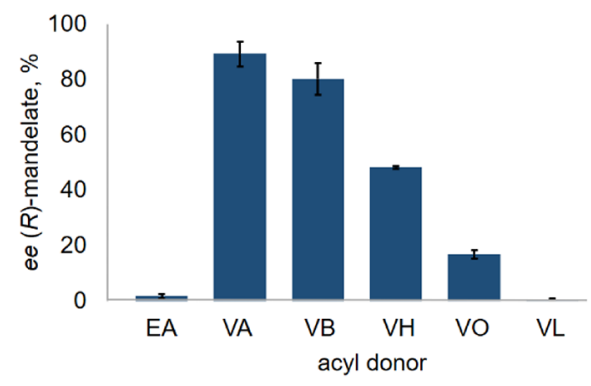

d)

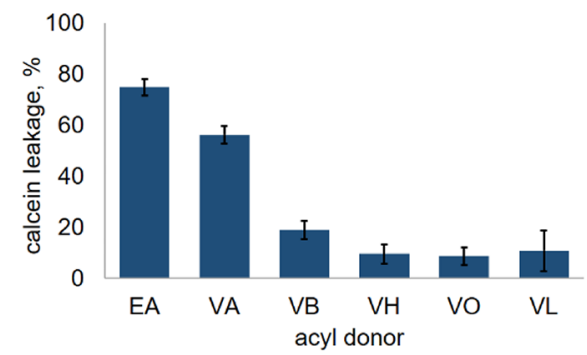

Figure 1. Effects of the acyl donor on MR activity $(a, b)$, conversion in the KR (c), and vesicle membrane integrity (d). ( $a, b)$ Remaining ee of $(R)$ mandelate after racemization by encapsulated MR in biphasic systems with $10 \%(\mathrm{v} / \mathrm{v}$ ) aqueous buffer and $90 \%$ (v/v) $n$-heptane as a function of vinyl acetate concentration (a) and type of acyl donor (at a constant concentration of $100 \mathrm{mM}$ ) (b). (c) Influence of the type of acyl donor (in each case at a concentration of $100 \mathrm{mM}$ in diisopropyl ether) on the conversion of $(R, S)$-mandelate during the KR. (d) Calcein leakage from loaded vesicles during incubation in aqueous buffer with $20 \%(\mathrm{v} / \mathrm{v})$ of the respective acyl donor. Here, no organic bulk phase was present. Abbreviations: EA, ethyl acetate; VA, vinyl acetate; VB, vinyl butyrate; VH, vinyl hexanoate; VO, vinyl octanoate; and VL, vinyl laurate. $N=3$.

In recent years, the concept of compartmentalization has been successfully used in different chemo- and multi-enzymatic reactions to overcome incompatibilities not only using macroscale compartments ${ }^{11-17}$ but also by the entrapment of catalysts in micro- or nanostructures. ${ }^{18-24}$ Artificial polymeric vesicles, so-called polymersomes, have proven to be very suitable nanocompartments for the spatial separation of incompatible enzymes in aqueous reaction systems. ${ }^{23}$ Vesicles made of poly(2-methyloxazoline $)_{15}$-poly(dimethylsiloxane $)_{68^{-}}$ poly(2-methyloxazoline) $)_{15}$ (PMOXA-PDMS-PMOXA) (Figure $\mathrm{S} 1$ ) are particularly suitable for this purpose as this polymer enables the functional integration of membrane proteins to establish a selective mass transport into and out of the nanocompartments. ${ }^{25,26}$ Notably, this type of polymer- some can also be used in biphasic reaction systems if macromolecules shall be retained inside the vesicles. Although the membrane is slightly permeabilized by organic solvents or ionic liquids, allowing small molecules like calcein $(0.6 \mathrm{kDa})$ to pass through, it is not completely disintegrated and the vesicles remain morphologically intact. ${ }^{22,27}$ Thus, entrapped enzymes can be shielded from the organic bulk phase to avoid denaturation by phase toxicity. The encaspulated $M R$, for example, remained active in different highly dispersed biphasic systems for more than $24 \mathrm{~h}$, whereas the free enzyme got completely inactivated within $1 \mathrm{~h}^{22}$ Therefore, polymeric vesicles might enable the application of lipases and solventsensitive isomerases in one pot. The aim of this study was to 
investigate this nanocompartmentalization approach on the example of the resolution of $(R, S)$-mandelate (Scheme 1$)$.

\section{MATERIALS AND METHODS}

Vesicle production. Vesicle production was done by an earlier published method using miniaturized stirred tank reactors. ${ }^{28}$ Detailed information on the polymer used can be found in the Supporting Information.

Calcein-Loaded Vesicles. For calcein-loaded vesicles, $0.1 \mathrm{M}$ Tris- $\mathrm{HCl}$ buffer ( $\mathrm{pH} 8.0$ ) with $15 \mathrm{mM}$ calcein was provided and $600 \mu \mathrm{L}$ of a $20 \%(\mathrm{w} / \mathrm{v})$ ethanolic polymer solution was injected under continuous stirring at $4000 \mathrm{rpm}$ using an S-type stirrer in unbaffled miniaturized stirred tanks (bioREACTOR48, 2mag, Munich, Germany). Thereby, a final polymer concentration of $1 \%(\mathrm{w} / \mathrm{v})$ in a final volume of $12 \mathrm{~mL}$ was reached. The process was continued at $25{ }^{\circ} \mathrm{C}$ until a polydispersity index $(\mathrm{PDI})<0.25$ was reached, as measured by dynamic light scattering.

MR-Loaded Vesicles. Expression, purification, and encapsulation of MR as well as the purification and concentration of the resulting vesicles were done as described previously. ${ }^{22,28,29}$ A detailed description of these methods can be found in the Supporting Information.

Catalytic Experiments in Two-Phase Systems. If not stated otherwise, all catalytic experiments and examinations on membrane integrity were performed at a $10 \mathrm{~mL}$ scale in miniaturized stirred tank reactors using the bioREACTOR 48 (2mag, Munich, Germany) with an S-type stirrer at $1000 \mathrm{rpm}$. The use of simple glass cylinders with cross-shaped magnetic stirrers at $500 \mathrm{rpm}$, as they were used for the initial experiments shown in Figure $1 \mathrm{~d}$ and Figure $\mathrm{S} 3$ in the Supporting Information, was insufficient to achieve an adequate dispersion when the solid lipase was applied together with the vesicle dispersion. To apply organic solvents in the bioREACTOR 48 system, the original single-use polystyrol vessels (2mag, Munich) had to be replaced by custom-made borosilicate glass vessels with identical dimensions.

For all experiments involving encapsulated $\mathrm{MR}$, the vesicle concentration was adjusted to $0.02 \%(\mathrm{w} / \mathrm{v})$ in the final reaction volume by injecting $100 \mu \mathrm{L}$ of a $2 \%(\mathrm{w} / \mathrm{v})$ stock solution in an aqueous buffer. If the final water proportion in the reactor had to be lower than $1 \%(\mathrm{v} / \mathrm{v})$, the vesicles were concentrated respectively by ultracentrifugation for $1 \mathrm{~h}$ at $55,000 g$

For all catalytic experiments in two-phase systems, a buffer containing $100 \mathrm{mM}$ 3-( $\mathrm{N}$-morpholino)propanesulfonic acid, $10 \mathrm{mM} \mathrm{NaCl}$, and $3.3 \mathrm{mM} \mathrm{MgCl}_{2}$ with $\mathrm{pH} 7.5$ was used as the aqueous phase.

After termination of the process, the organic solvent was evaporated by surface gassing with nitrogen and the sample was then dried for $24 \mathrm{~h}$ at $60{ }^{\circ} \mathrm{C}$ prior to preparation for highperformance liquid chromatography (HPLC) measurements.

Racemization. All racemization experiments were done for $24 \mathrm{~h}$ at $25{ }^{\circ} \mathrm{C}$. The reactors were filled with the respective organic solvent containing dissolved $(R)$-mandelate before the acyl donor and the aqueous buffer were added. Subsequently, the resulting two-phase system was stirred for $10 \mathrm{~min}$ before the vesicle dispersion was added through the sampling hole of the reactor under sustained stirring. Hereby, a high dispersion of the system was guaranteed before the catalyst was added.

Kinetic Resolution. For each reaction, $70 \mathrm{mg}$ of BCL was provided as a lyophilized powder $\left(>30,000 \mathrm{U} \mathrm{g}^{-1}\right.$, product number 54327) or immobilized on Immobead 150 (>900 U $\mathrm{g}^{-1}$, product number 534641), both purchased from SigmaAldrich (Taufkirchen, Germany). Afterward, the respective amount of organic solvent, containing dissolved $(R, S)$ mandelate, and the respective acyl donor were added. The aqueous buffer was added directly after the reactor was started through the sampling hole under sustained stirring. All kinetic resolution experiments were done for $72 \mathrm{~h}$ at $25^{\circ} \mathrm{C}$.

One-Pot Kinetic Resolution with Re-racemization (Final Executed Process). In the first $48 \mathrm{~h}$, the kinetic resolution of 1 $\mathrm{mM}(R, S)$-mandelate took place using $70 \mathrm{mg}$ immobilized lipase in diisopropyl ether supplemented with $150 \mathrm{mM}$ vinyl laurate and $0.1 \%$ (v/v) aqueous buffer. Subsequently, the lipase was removed by filtration using a $5 \mu \mathrm{m}$ nylon syringe filter. The following racemization was started by the addition of the vesicle dispersion as described for the racemization experiments, and the aqueous phase was increased to $1.0 \%(\mathrm{v} / \mathrm{v})$. After an additional $24 \mathrm{~h}$, the vinyl laurate concentration was increased to $750 \mathrm{mM}$ and $70 \mathrm{mg}$ of immobilized lipase was added. After $48 \mathrm{~h}$, the reaction was terminated, resulting in an overall process time of $120 \mathrm{~h}$.

Analytics. Determination of Vesicle Concentration. Concentration measurements were done as optical densitiy (OD) at $350 \mathrm{~nm}$ in 96-well microtiter plates containing $100 \mu \mathrm{L}$ sample volume using an Infinite $\mathrm{M}$ Nano+ (TECAN, Männedorf, Switzerland). The correlation between optical density and polymer concentration was linear up to OD 0.5 and was determined by a serial dilution of vesicle samples with a known polymer concentration. The correlation is $c$ (polymer, $\%(\mathrm{w} / \mathrm{v}))=1.44 \times \mathrm{OD}_{350}$.

Static Light Scattering (SLS). The aggregation number $N_{\text {Agg. }}$ of the produced vesicles was determined by static light scattering (SLS) at vesicle concentrations of $0.05 \%(\mathrm{w} / \mathrm{v})$, $0.1 \%(\mathrm{w} / \mathrm{v}), 0.167 \%(\mathrm{w} / \mathrm{v})$, and $0.2 \%(\mathrm{w} / \mathrm{v})$ using an ALV/ CGS-3 compact goniometer equipped with a diode laser with $50 \mathrm{~mW}$ at $660 \mathrm{~nm}$. Measurements were executed at $26^{\circ} \mathrm{C}$ with detection angles from 20 to $150^{\circ}$ ( $5^{\circ}$ steps). The refractive index increment was assumed as $0.188 \mathrm{~mL} \mathrm{~g}^{-1}$, as it was done for comparable vesicles. ${ }^{30}$ Molecular mass, gyradius, and second virial coefficient were determined using a secondorder Berry plot. SLS was done as an order measurement by ALV (Langen, Germany).

Vesicles for SLS measurements were prepared as described by Poschenrieder et al. in $\mathrm{ddH}_{2} \mathrm{O}$ and extruded three times using a $0.2 \mu \mathrm{m}$ unipore PC syringe filter. ${ }^{28}$

Dynamic Light Scattering (DLS). The quality of the vesicles was controlled by DLS using a Zetasizer NS (Malvern, Worcestershire, UK) as reported elsewhere. ${ }^{28}$

The formation of mandelate micelles was investigated by DLS using an experimental setup as described by Piszko et al. ${ }^{31}$ Here, the samples with $(R, S)$-mandelate concentrations of 0.5 , 2,5 , and $10 \mathrm{mM}$ in diisopropyl ether were filled in glass cuvettes. Diisopropyl ether was filtered to reduce the influence of possible particles. The measurements were performed at a temperature of $25.05{ }^{\circ} \mathrm{C}$ and a scattering angle of $90^{\circ}$. As a light source, a fiber laser (Azurlight Systems) operated on a single longitudinal mode with a laser wavelength of $532 \mathrm{~nm}$ and an incident laser power of $196 \mathrm{~mW}$ was used. For the continuous phase, refractive index and dynamic viscosity of diisopropyl ether were taken from Meng et al. ${ }^{32}$

Calcein Leakage. Calcein leakage was determined by fluorescence spectroscopy ${ }^{22}$ using an Infinite Nano+ (TECAN) at an excitation of $490 \mathrm{~nm}$ and an emission of $537 \mathrm{~nm}$ in 96-well fluorescence microtiter plates with a sample 
volume of $100 \mu \mathrm{L}$. Prior to this, vesicles were removed at $80,000 \mathrm{~g}$ for $1 \mathrm{~h}$ and samples were taken from the supernatant. If samples were taken from two-phase systems, phase separation was done at $16,000 \mathrm{rpm}$ for $10 \mathrm{~min}$ to remove the organic solvent prior to ultracentrifugation. In this case, partition coefficients of calcein were determined for all applied two-phase systems to ensure correct calcein quantification. The maximal possible loss of calcein was determined by solubilization of the vesicles using $1 \%(\mathrm{v} / \mathrm{v})$ n-octylpolyoxyethylene (BACHEM, Bubendorf BL, Switzerland) and subsequent measurement of the released calcein. This has already been shown to be a reliable method since the vesicles are completely disintegrated at this detergent concentration. ${ }^{33}$

HPLC. All samples were resuspended in 2-propanol and filtered through $0.22 \mu \mathrm{m}$ nylon syringe filters prior to HPLC measurements. Since vinyl laurate cannot be evaporated and shows very low solubility for mandelate, the amount of 2 propanol added depends on the vinyl laurate concentration used in the reaction. Here, we used a 2-propanol to vinyl laurate proportion of $1: 1$.

HPLC measurements were done with a chiral Lux $3 \mu \mathrm{m}$ Cellulose-1 column, purchased from Phenomenex (Torrance, CA, USA), at a flow rate of $0.4 \mathrm{~mL} \mathrm{~min}^{-1}$ at $30{ }^{\circ} \mathrm{C}$ in $90 \%(\mathrm{v} /$ v) $n$-hexane and $10 \%(\mathrm{v} / \mathrm{v}) 2$-propanol modified with $0.4 \%$ trifluoroacetic acid. For experiments using vinyl octanoate or vinyl laurate as the acyl donor, the $n$-hexane concentration was increased to $94 \%(\mathrm{v} / \mathrm{v})$. Detection occurred at $258 \mathrm{~nm}$. The injection volume for racemization experiments was $10 \mu \mathrm{L}$. For experiments on kinetic resolution, the injection volume varied between 5 and $30 \mu \mathrm{L}$, depending of the dilution during the sample preparation, since samples containing higher vinyl laurate concentrations had to be diluted with 2-propanol to avoid solidification.

Identification and quantification of $(R)$-mandelate, $(S)$ mandelate, and the acylated products were done by a serial dilution of the pure compounds.

Circular Dichroism (CD) Spectroscopy. CD spectroscopy was used for activity determination of $\mathrm{MR}$ in pure aqueous systems to study the influence of dissolved acyl donors, $n$ heptane, toluene, and dissopropyl ether. Measurements were done according to the previously described method using a J815 CD-spectrometer equipped with a PTC-4235/15 controller and the Spectra Manager software from Jasco (Pfungstadt, Germany). ${ }^{22}$ Measurement settings were as follows: time course measurement, wavelength $268 \mathrm{~nm}$, band width $1 \mathrm{~nm}$, and data pitch $1 \mathrm{~s}$. Enzyme activity was calculated from the initial slope of the CD signal at the racemization of 50 $\mathrm{mM}$ enantiopure $(R)$-mandelate in the following buffer: 10 $\mathrm{mM}$ 3-(N-motpholino)propanesulfonic acid, $10 \mathrm{mM} \mathrm{NaCl}$, and $3.3 \mathrm{mM} \mathrm{MgCl}_{2}$ with $\mathrm{pH}$ 7.5. All measurements were done in a $1 \mathrm{~mL}$ UV quartz glass (QS) micro cell (Hellma Analytics, Munich, Germany).

$M R$ Concentration. MR concentration was determined with a Pierce BCA Protein Assay Kit (Thermo Scientific, Waltham MA, USA) as described by the manufacturer's protocol.

\section{RESULTS AND DISCUSSION}

Kinetic Resolution. To achieve high yields in the KR by the selective $O$-acylation of $(S)$-mandelate, the influence of the excess of acyl donor and the water proportion in the biphasic system was characterized. Vinyl acetate was initially chosen as the acyl donor since it has high solubility in most organic solvents and has already been used successfully in KRs with diverse lipases, including the model system studied here. ${ }^{34,35}$ Since the conversions were comparable with $n$-heptane, toluene, and diisopropyl ether as the bulk organic phase (Figure S3a), n-heptane was chosen because of its low volatility, low toxicity, and low impact on the membrane integrity of the polymeric vesicles. ${ }^{22}$ With water proportions of up to $0.1 \%(\mathrm{v} / \mathrm{v})$, a 20 -fold excess of vinyl acetate over $(S)$ mandelate was necessary to approximately reach the maximum yield of $50 \%$ (here: $47.4 \%$ ). Higher water proportions led to a rapid decrease in yield (Figure S3b,c).

Racemization. Before investigating racemization under the conditions set by the BCL, the MR was encapsulated as described recently. ${ }^{22,33}$ The generated vesicles had a numberbased mean diameter of $123 \mathrm{~nm}$ with on average 7.2 encapsulated MR subunits or 0.9 complete molecules of the homooctameric enzyme. The vesicle concentration was $0.02 \%$ $(\mathrm{w} / \mathrm{v})$ in the final reaction volume, corresponding to an enzyme concentration of $3.9 \mu \mathrm{g} \mathrm{mL}{ }^{-1}$. For all racemization experiments, enantiopure $(R)$-mandelate was applied as the substrate and the remaining enantiomeric excess (ee) was measured after $24 \mathrm{~h}$.

Acyl Donors. As vinyl acetate inactivates the $\mathrm{MR},{ }^{9}$ its impact on the encapsulated enzyme was evaluated. To keep the effect of the organic bulk phase as low as possible, $n$-heptane with $10 \%(\mathrm{v} / \mathrm{v})$ aqueous phase was used since the reduction of MR activity by molecular toxicity was $<8 \%$ for dissolved $n$-heptane and therefore quite low compared to other organic solvents (Figure S4). The high ee values measured even at low vinyl acetate concentrations indicated a rapid deactivation of the enzyme (Figure 1a).

Accordingly, encapsulated MR is also strongly influenced by small amounts of the acyl donor. This can be explained by the comparatively high water solubility of vinyl acetate with an octanol-water partition coefficient $(\log P)$ of 0.7 and its membrane permeabilizing effect on the vesicles, as shown in membrane integrity experiments in Figure 1d. Thus, vinyl acetate can easily pass the vesicle membrane and reach the aqueous core. In view of the need for a 20 -fold excess of acyl donor for the $\mathrm{KR}$, it became clear that vinyl acetate cannot be used for this reaction system.

To exclude the inhibiting acyl donor from the aqueous vesicle lumen, more hydrophobic vinyl esters with longer hydrocarbon chains were tested. The remaining ee of $(R)$ mandelate indeed decreased with increasing length of the acyl donor, indicating less MR inactivation (Figure 1b). Notably, no MR inactivation was observed for vinyl laurate. We investigated whether the decreasing inactivation was caused by the increasing hydrophobicity of the donor, meaning lower water solubility and lower membrane permeabilization, or by the increasing steric exclusion from the active site of the enzyme. In pure aqueous buffer, $2.8 \mathrm{mM}$ vinyl acetate, butyrate, or hexanoate was dissolved (solubility limit of vinyl hexanoate in water at $25^{\circ} \mathrm{C}$ ). No difference in MR activity was observed, indicating no impact of the donor size. Therefore, the exclusion of the acyl donor from the aqueous phase respectively the lower membrane permeabilization seem to be the main factors that protect the MR from inactivation. The comparison of MR inactivation by vinyl acetate and its hydrogenated counterpart ethyl acetate clearly shows that the vinyl group causes the inactivation (Figure 1b). Ethyl acetate, however, could not be used for the BCL reaction since the product yield was $<5 \%$ after $72 \mathrm{~h}$. For the reactive enol esters, the maximum achievable conversion of $50 \%$ was almost 
a)

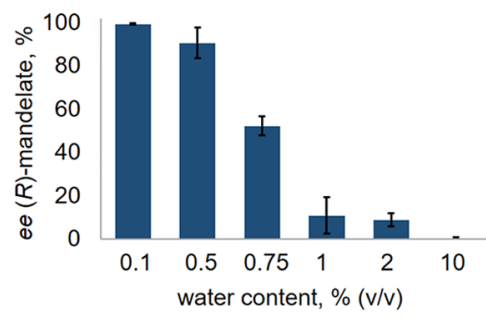

b)

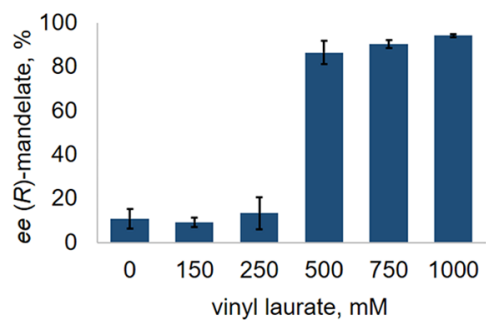

c)

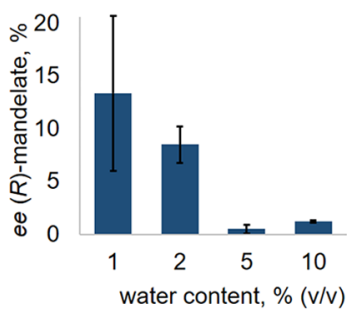

Figure 2. Effect of the water content of the racemization of $(R)$-mandelate in biphasic systems with diisopropyl ether as the organic bulk phase. The remaining enantiomeric excess of $(R)$-mandelate after $24 \mathrm{~h}$ is shown. (a) Effect of the water proportion in the absence of the acyl donor. (b) Effect of different vinyl laurate concentrations at a constant water content of $1 \%(\mathrm{v} / \mathrm{v})$. (c) Effect of different water contents at a constant vinyl laurate concentration of $250 \mathrm{mM}$. $N=3$.

reached for all donors (Figure 1c). Furthermore, for all enol esters, the ee was $>99.9 \%$ after the complete conversion of $(S)$ mandelate. However, if the process was continued after $(S)$ mandelate was used up, the ee decreased for vinyl acetate, reaching $96 \%$ after 7 additional days, but remained constant for all other acyl donors. It is known that larger acyl donors can lead to higher optical purity caused by steric effects at the active site of the enzyme. This has been also shown for the BCL-catalyzed transesterification of $(R, S)$-1-phenylethanol, where the enantioselectivity was $E>200$ for vinyl laurate and $E=148$ for vinyl acetate. ${ }^{36}$

Vesicle Membrane Integrity. In addition to the inactivation of $\mathrm{MR}$, we also observed polymer agglomeration in the presence of vinyl acetate, indicating vesicle disintegration. A calcein leakage experiment, which is a well-established method to detect even small defects in polymeric membranes, ${ }^{37,38}$ revealed a correlation between molecule release and donor hydrophilicity, decreasing from ethyl acetate (75\%) to vinyl butyrate (19\%). For the acyl donors with even larger hydrocarbon chains, molecule loss was almost constant between 9 and $11 \%$ (Figure 1d). Since vinyl laurate accordingly not only avoids MR inactivation and increases the optical purity in the KR but also shows less influence on the vesicle integrity, it was used in all further experiments.

Substrate Transfer. Because the solubility of the polar mandelate in nonpolar $n$-heptane $(\log P 4.3)$ was significantly increased by the addition of vinyl acetate $(\log P 0.4)$, a switch to the hydrophobic vinyl laurate $(\log P 5.8)$ also required the change of the organic bulk phase to the more polar diisopropyl ether $(\log P 1.5)$, which is also suitable in terms of BCL and MR activity (Figures S3a and S4). Although $10 \mathrm{mM}(R, S)$ mandelate in diisopropyl ether appeared as a clear-transparent solution, mandelate micelles were detected via dynamic light scattering. Their hydrodynamic diameters could be estimated to be in the range between 1 and $3 \mu \mathrm{m}$. Consequently, no mass transfer from the organic into the aqueous phase occurred, resulting in no measurable $\mathrm{MR}$ activity. In all further experiments, the mandelate concentration was lowered to 1 $\mathrm{mM}$ where only a weak signal rising from translational micelle diffusion could be obtained. The acylation reaction, however, was unaffected by the formation of mandelate micelles.

Water Proportion. The determination of the minimum water proportion for the MR reaction revealed that at least $1 \%$ $(\mathrm{v} / \mathrm{v})$ is needed for sufficient racemization (maximum ee of $10 \%$, Figure $2 \mathrm{a}$ ). Although diisopropyl ether molecules in the aqueous phase reduce the MR activity by $32 \%$ (Figure $S 4$ ), this does not explain the low conversions at water contents below $1 \%(\mathrm{v} / \mathrm{v})$. Calcein leakage experiments were used again to investigate if this is caused by vesicle damage. Although the membrane integrity correlated with the applied organic bulk phase, increasing from $n$-heptane via toluene to diisopropyl ether, it was independent of the water proportion ranging from 3 to $80 \%(\mathrm{v} / \mathrm{v})$ (Figure S6). However, water proportions of $<3 \%(\mathrm{v} / \mathrm{v})$ cannot be reliably investigated in this analytical setup, but still some assumptions can be made: The polymeric vesicles can adsorb permanently at the interphase forming a Pickering emulsion. ${ }^{22,39}$ Thus, once adsorbed, no increased contact frequency of the vesicles should occur by an increasing interphase or different phase proportions. This might explain the observed independence of membrane integrity from phase proportion and degree of dispersion. Anyhow, at water proportions below $1 \%(\mathrm{v} / \mathrm{v})$, the vesicles agglomerated. Probably, the interfacial area became limiting, resulting in increased vesicle interaction. Since only vesicles at the agglomerate surface are in contact with the interphase, the inner vesicles are subjected to mass transport limitations, which could explain the poor MR activity at very low water contents (Figure 2a). Yang et al. ${ }^{40}$ also showed that the specific activity of enzymes in the aqueous phase of a hexane/water system is 8 times higher if a Pickering emulsion is formed by the addition of microparticles, leading to an increased interfacial area. If the enzyme is additionally encapsulated in the microparticles, accumulating directly at the interphase, the activity is again increased by a factor of 1.5 due to better substrate supply.

Combined Effect of Water Proportion and Acyl Donor. So far, we examined the impact of water proportions and acyl donors on the MR reaction, showing the necessity of at least $1 \%(\mathrm{v} / \mathrm{v})$ aqueous phase and the preferable use of the hydrophobic acyl donor vinyl laurate. To study the combined impact of both, different acyl donor concentrations were used at the most promising water proportion of $1 \%(\mathrm{v} / \mathrm{v})$ with diisopropyl ether as the bulk phase (Figure $2 \mathrm{~b}$ ). Vinyl laurate concentrations of up to $250 \mathrm{mM}$ have no additional impact on the MR reaction, still yielding an ee of around $10 \%$. For higher concentrations, the ee strongly increases to $80-90 \%$, indicating the inexpediency of these concentrations. Comparing the racemization in the absence of vinyl laurate (Figure $2 \mathrm{a}$ ) and in the presence of $250 \mathrm{mM}$ vinyl laurate (Figure 2c), the influence of the water proportion is similar for both conditions. At $1 \%(\mathrm{v} / \mathrm{v})$ aqueous phase, the ee is $11 \%$ without and $13 \%$ with vinyl laurate. At $2 \%(\mathrm{v} / \mathrm{v})$ aqueous phase, the $e e$ is actually identical for both conditions (9\%). Thus, a faster MR deactivation by the combined effect of small water proportions and the acyl donor was excluded. 
Process Stability. For the one-pot deracemization, encapsulated MR and particulate BCL powder had to be applied in the same reaction vessel. However, for water proportions $<2 \%(\mathrm{v} / \mathrm{v})$, lipase powder and vesicles agglomerated gradually. After $12 \mathrm{~h}$, the entire reactor content had agglomerated, forming a single cluster of polymer and enzymes with complete loss of activity for both catalysts. In contrast, with only one of the two components, no agglomeration occurred even at water proportions <2\% (v/v) (Figure 3).

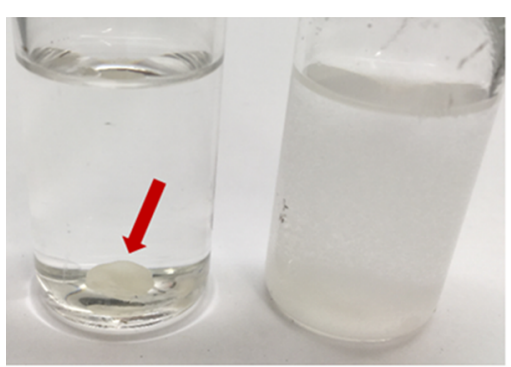

Figure 3. Agglomeration of polymeric vesicles and lyophilized lipase at water contents $<2 \%(\mathrm{v} / \mathrm{v})$ (left). No agglomeration was observed for the use of lipase without vesicles (right) or vesicles without lipase (not shown).

To avoid agglomeration, the particle size of the lipase was increased by using the carrier Immobead 150, which is commercially available with covalently immobilized BCL. The spherical carriers consist of cross-linked methacrylates with diameters of 150 to $300 \mu \mathrm{m}$. Using this carrier, no agglomeration occurred and enzyme inactivation was completely avoided. Thus, Immobead 150 was used for all experiments containing lipases.

Deracemization in One Pot. Since the MR narrows the potential process conditions for the one-pot reaction, a detailed analysis of the requirements for the KR was necessary (Figure 4a). While $150 \mathrm{mM}$ vinyl laurate was sufficient to achieve the maximum yield of $50 \%$ at $0.1 \%(\mathrm{v} / \mathrm{v})$ water content, the required amount of acyl donor increased significantly with increasing water proportions. Considering that at least $1 \%(\mathrm{v} / \mathrm{v})$ aqueous phase with a maximum of 250 $\mathrm{mM}$ vinyl laurate is necessary for the racemization reaction to run almost completely, the simultaneous execution of racemization and acylation is not possible since $750 \mathrm{mM}$ vinyl laurate would be required at $1 \%(\mathrm{v} / \mathrm{v})$ aqueous phase.

On closer examination, however, a process route for the combined reaction of $\mathrm{BCL}$ and $\mathrm{MR}$ in one pot was identified (Figure $4 \mathrm{~b})$. (A) Starting with $0.1 \%(\mathrm{v} / \mathrm{v})$ aqueous phase, 150 $\mathrm{mM}$ vinyl laurate is sufficient to completely convert the $(S)$ mandelate. (B) Afterward, the water proportion has to be increased to $1 \%(\mathrm{v} / \mathrm{v})$ for the racemization, achieving an $e e$ of 9.2\% as shown in Figure 2b. Since the lipase would catalyze the reverse reaction under those conditions, it was removed before via filtration. (C) As a final step, the vinyl laurate concentration is increased to $750 \mathrm{mM}$ and fresh lipase is added, converting the newly formed $(S)$-mandelate. Considering the ee of $9.2 \%$ after the racemization and the complete conversion during the two KR steps, a product yield of $72.7 \%$ is theoretically possible. a)

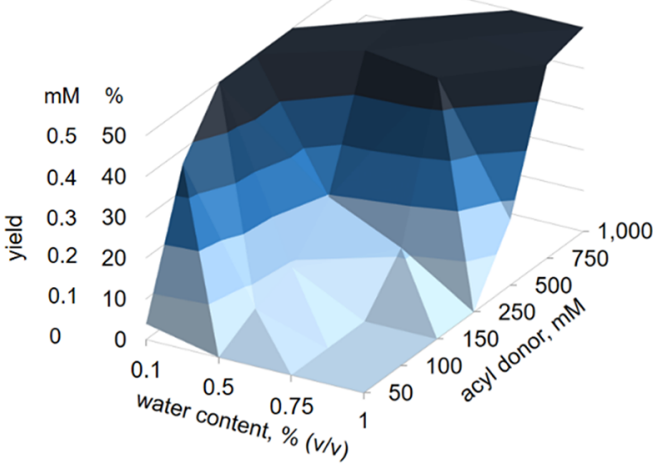

c)

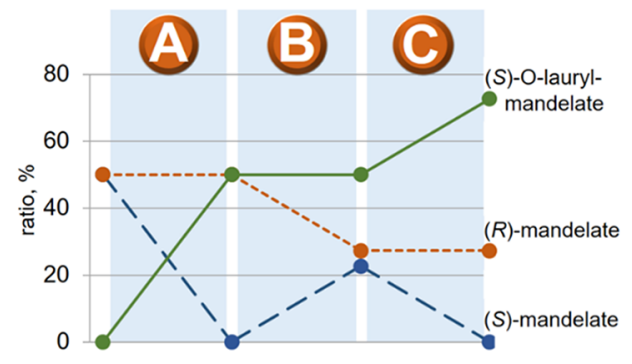

b)

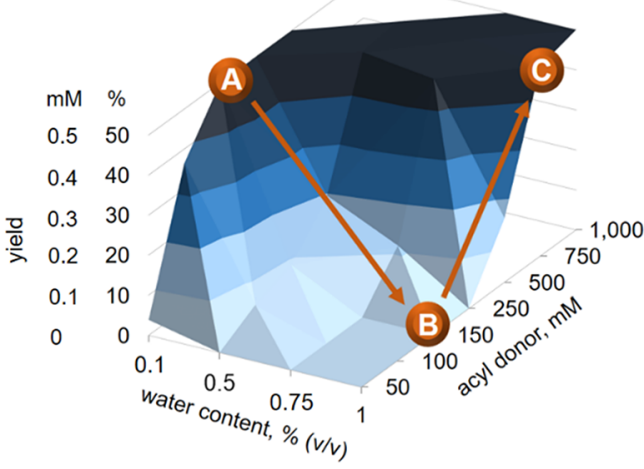

d)

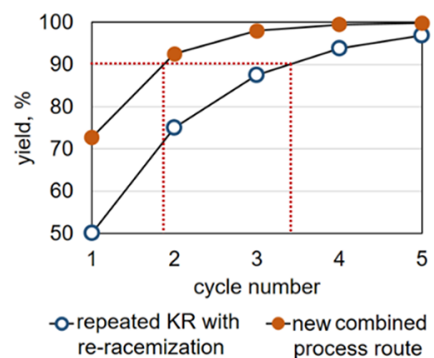

Figure 4. (a) Influence of water proportion and vinyl laurate concentration on the yield of $(S)$-O-lauryl mandelate in the KR of $(R, S)$-mandelate catalyzed by immobilized BCL. Each data point is representing three independent experiments. Standard deviations for data points were between 0.0 and 3.81. (b) Arrows indicate the possible process route for the one-pot reaction of BCL and MR: (A) conditions of the first KR, (B) conditions of the re-racemization, and $(\mathrm{C})$ conditions of the second KR. A detailed description of the process route is found in the main text. (c) Gradient of the reactants $(R)$ - and $(S)$-mandelate as well as the product $(S)$-O-lauryl mandelate during the single process steps as they were described in (b). (d) Comparison of the achievable yield in a classic kinetic resolution with the here presented new concept. Each cycle represents one deracemization step. 
Indeed, performing the reaction route as described, we were able to yield $72 \%$ of enantiopure $(S)$-O-lauryl mandelate, no measurable $(S)$-mandelate, and $28 \%(R)$-mandelate after an overall reaction time of $120 \mathrm{~h}$, being respectively $48 \mathrm{~h}$ for the $\mathrm{KR}$ steps and $24 \mathrm{~h}$ for the racemization step. It is important to note that the individual reaction steps were not optimized with respect to the minimum time required. Long reaction times were chosen to exclude the factor time as a cause for incomplete conversions. Preliminary calculations on the potential to increase the space-time yield showed that it is possible to shorten the entire process time from 120 to $48 \mathrm{~h}$ or even less.

The course of the ratio of the reactants $(R)$ - and $(S)$ mandelate and the product $(S)$-O-lauryl mandelate after each step is shown in Figure 4c. Although the lipase had to be removed prior to the racemization, no additional operations such as solvent switching, product removal, or acyl donor removal were necessary, allowing the process to be done in a one-pot reaction without costly purification steps. Additionally, if higher yields than $72 \%$ are required, the number of purification cycles is significantly reduced compared to the classic concept of repeated $\mathrm{KR}$ with re-racemization (Figure 4d). While four cycles with three intermediate purification steps are required to obtain a yield $>90 \%$ with this concept, only two cycles with one intermediate purification step are needed for the new combined process route presented here (dotted line, Figure 4d). For an eco-efficient process, the reuse of the unreacted acyl donor after each cycle is of the utmost importance, but due to the different physicochemical properties of the molecules in the reaction mixture, separation and reuse should be relatively easy to implement. The isolation of the product and the reuse of the unreacted acyl donor will be investigated in future studies following an optimization of the space-time yield and the scale-up from the miniaturized stirred tank reactors used in this study to reactors on a liter scale. The excellent scalability of processes that were developed in the bioREACTOR 48 system has already been demonstrated many times. ${ }^{41-43}$

\section{CONCLUSIONS}

In summary, we performed a deracemization of $(R, S)$ mandelate, yielding $72 \%$ of the enantiopure product $(S)-O$ lauryl mandelate and $28 \%$ of the unreacted $(R)$-mandelate. The reaction was done in a single reaction vessel by combining two enzymes with oppositional needs regarding their reaction environment. Polymeric vesicles facilitated the combination of the per se incompatible biocatalysts by their spatial separation in biphasic systems with high phase proportions of the organic solvent. Due to the formation of substrate micelles in the applied organic solvent, which prevented the mass transfer of the substrate from the organic bulk phase into the aqueous phase containing the isomerase, the substrate concentration was limited to $1 \mathrm{mM}$. Since the formation of substrate micelles in organic solvents is certainly to be assessed as an exception, the application of the nanocompartmentalization approach to other deracemization reactions involving much higher substrate concentrations should be feasible.

\section{ASSOCIATED CONTENT}

\section{(s) Supporting Information}

The Supporting Information is available free of charge at https://pubs.acs.org/doi/10.1021/acsomega.1c04694.
Additional Materials and Methods section containing information about the polymer used for vesicle formation as well as expression, purification, and entrapment of mandelate racemase and the purification and concentration of the resulting vesicles; additional Results and Discussion section containing the vesicle characterization via DLS and SLS, characterization of mandelate micelles via DLS, initial experiments for the $\mathrm{KR}$ of $(R, S)$-mandelate, impact of the molecular toxicity of organic solvents on the $\mathrm{MR}$, investigation of an alternative lipase (CALB) or various acyl donors, impact of the reaction conditions on vesicle membrane integrity, and HPLC-chromatogram and retention times for the identification and quantification of the used compounds (PDF)

\section{AUTHOR INFORMATION}

\section{Corresponding Author}

Kathrin Castiglione - Department of Chemical and Biological Engineering, Institute of Bioprocess Engineering, FriedrichAlexander University Erlangen-Nürnberg, 91052 Erlangen, Germany; 이이이.org/0000-0001-9086-0036;

Email: kathrin.castiglione@fau.de

\section{Authors}

Florian Golombek - Department of Chemical and Biological Engineering, Institute of Bioprocess Engineering, FriedrichAlexander University Erlangen-Nürnberg, 91052 Erlangen, Germany

Marco Haumann - Department Chemie- und Bioingenieurwesen, Lehrstuhl für Chemische Reaktionstechnik (CRT), Friedrich-Alexander Universität Erlangen-Nürnberg (FAU), Erlangen 91058, Germany; 이이이.org/00000002-3896-365X

Matthias S.G. Knoll - Department of Chemical and Biological Engineering, Institute of Advanced Optical Technologies Thermophysical Properties, Friedrich-Alexander University Erlangen-Nürnberg, Erlangen 91052, Germany; Erlangen Graduate School of Advanced Optical Technologies (SAOT), 91052 Erlangen, Germany

Andreas Paul Fröba - Department of Chemical and Biological Engineering, Institute of Advanced Optical Technologies Thermophysical Properties, Friedrich-Alexander University Erlangen-Nürnberg, Erlangen 91052, Germany; Erlangen Graduate School of Advanced Optical Technologies (SAOT), 91052 Erlangen, Germany

Complete contact information is available at: https://pubs.acs.org/10.1021/acsomega.1c04694

\section{Notes}

The authors declare no competing financial interest.

\section{ACKNOWLEDGMENTS}

The authors gratefully acknowledge the financial support from the German Federal Ministry of Education and Research (Grant 031B0221) and the Erlangen Graduate School in Advanced Optical Technologies (SAOT) by the Bavarian State Ministry for Science and Art. We thank Daniela Egger, Marina Raab, Johanna Gogl, and Christine Schülein for their technical assistance and Andreas Perlick for helpful discussions. 


\section{REFERENCES}

(1) Verho, O.; Bäckvall, J.-E. Chemoenzymatic dynamic kinetic resolution: a powerful tool for the preparation of enantiomerically pure alcohols and amines. J. Am. Chem. Soc. 2015, 137, 3996-4009.

(2) Diaz-Rodriguez, A.; Lavandera, I.; Gotor, V. Why Leave a Job Half Done? Recent Progress in Enzymatic Deracemizations. Curr. Green Chem. 2015, 2, 192-211.

(3) Felfer, U.; Kroutil, W.; Strauss, U. T.; Faber, K. Biocatalytic Strategies for the Preparation of Chiral Building Blocks in 100\% Chemical and Optical Yield from Racemates. The 2nd International Electronic Conference on Synthetic Organic Chemistry 1998, 1667.

(4) Musa, M. M.; Hollmann, F.; Mutti, F. G. Synthesis of enantiomerically pure alcohols and amines via biocatalytic deracemisation methods. Catal. Sci. Technol. 2019, 9, 5487-5503.

(5) Schmidt, S.; Castiglione, K.; Kourist, R. Overcoming the Incompatibility Challenge in Chemoenzymatic and Multi-Catalytic Cascade Reactions. Chemistry 2018, 24, 1755-1768.

(6) Lafaquière, V.; Barbe, S.; Puech-Guenot, S.; Guieysse, D.; Cortés, J.; Monsan, P.; Simeon, T.; André, I.; Simeon, M. R. Control of Lipase Enantioselectivity by Engineering the Substrate Binding Site and Access Channel. ChemBioChem 2009, 10, 2760-2771.

(7) May, O.; Nguyen, P. T.; Arnold, F. H. Inverting enantioselectivity by directed evolution of hydantoinase for improved production of L-methionine. Nat. Biotechnol. 2000, 18, 317-320.

(8) Verger, R. 'Interfacial activation' of lipases: Facts and artifacts. Trends Biotechnol. 1997, 15, 32-38.

(9) Kaftzik, N.; Kroutil, W.; Faber, K.; Kragl, U. Mandelate racemase activity in ionic liquids: scopes and limitations. J. Mol. Catal. A: Chem. 2004, 214, 107-112.

(10) Choi, W. J.; Lee, K. Y.; Kang, S. H.; Lee, S. B. Biocatalytic enantioconvergent separation of racemic mandelic acid. Sep. Purif. Technol. 2007, 53, 178-182.

(11) Sperl, J. M.; Carsten, J. M.; Guterl, J. -K.; Lommes, P.; Sieber, V. Reaction Design for the Compartmented Combination of Heterogeneous and Enzyme Catalysis. ACS Catal. 2016, 6, 63296334.

(12) Fink, M. J.; Schön, M.; Rudroff, F.; Schnürch, M.; Mihovilovic, M. D. Single Operation Stereoselective Synthesis of Aerangis Lactones: Combining Continuous Flow Hydrogenation and Biocatalysts in a Chemoenzymatic Sequence. Chem CatChem 2013, 5, 724-727.

(13) Heidlindemann, M.; Rulli, G.; Berkessel, A.; Hummel, W.; Gröger, H. Combination of Asymmetric Organo- and Biocatalytic Reactions in Organic Media Using Immobilized Catalysts in Different Compartments. ACS Catal. 2014, 4, 1099-1103.

(14) Wedde, S.; Rommelmann, P.; Scherkus, C.; Schmidt, S.; Bornscheuer, U. T.; Liese, A.; Gröger, H. An alternative approach towards poly- $\varepsilon$-caprolactone through a chemoenzymatic synthesis: combined hydrogenation, bio-oxidations and polymerization without the isolation of intermediates. Green Chem. 2017, 19, 1286-1290.

(15) Sato, H.; Hummel, W.; Gröger, H. Cooperative Catalysis of Noncompatible Catalysts through Compartmentalization: Wacker Oxidation and Enzymatic Reduction in a One-Pot Process in Aqueous Media. Angew. Chem., Int. Ed. 2015, 54, 4488-4492.

(16) Mwangi, M. T.; Runge, M. B.; Hoak, K. M.; Schulz, M. D.; Bowden, N. B. A materials approach to site-isolation of Grubbs catalysts from incompatible solvents and $m$-chloroperoxybenzoic acid. Chem.-Eur. J. 2008, 14, 6780-6788.

(17) Latham, J.; Henry, J.-M.; Sharif, H. H.; Menon, B. R. K.; Shepherd, S. A.; Greaney, M. F.; Micklefield, J. Integrated catalysis opens new arylation pathways via regiodivergent enzymatic $\mathrm{C}-\mathrm{H}$ activation. Nat. Commun. 2016, 7, 11873.

(18) Patterson, D. P.; Schwarz, B.; Waters, R. S.; Gedeon, T.; Douglas, T. Encapsulation of an Enzyme Cascade within the Bacteriophage P22 Virus-Like Particle. ACS Chem. Biol. 2014, 9, 359-365.

(19) Zhang, L.; Shi, J.; Jiang, Z.; Jiang, Y.; Qiao, S.; Li, J.; Wang, R.; Meng, R.; Zhu, Y.; Zheng, Y. Bioinspired preparation of polydop- amine microcapsule for multienzyme system construction. Green Chem. 2011, 13, 300-306.

(20) Azuma, Y.; Zschoche, R.; Tinzl, M.; Hilvert, D. Quantitative Packaging of Active Enzymes into a Protein Cage. Angew. Chem., Int. Ed. 2016, 55, 1531-1534.

(21) Uhrich, D.; von Langermann, J. Preparation and Characterization of Enzyme Compartments in UV-Cured Polyurethane-Based Materials and Their Application in Enzymatic Reactions. Front. Microbiol. 2017, 8, 2111.

(22) Golombek, F.; Castiglione, K. Polymersomes as Nanoreactors Enabling the Application of Solvent-Sensitive Enzymes in Different Biphasic Reaction Setups. Biotechnol. J. 2020, 15, 1900561.

(23) Klermund, L.; Poschenrieder, S. T.; Castiglione, K. Biocatalysis in Polymersomes: Improving Multienzyme Cascades with Incompatible Reaction Steps by Compartmentalization. ACS Catal. 2017, 7, $3900-3904$.

(24) Karume, I.; Musa, M. M.; Bsharat, O.; Takahashi, M.; Hamdan, S. M.; El Ali, B. Dual enzymatic dynamic kinetic resolution by Thermoanaerobacter ethanolicus secondary alcohol dehydrogenase and Candida antarctica lipase B. RSC Adv. 2016, 6, 96616-96622.

(25) Palivan, C. G.; Goers, R.; Najer, A.; Zhang, X.; Car, A.; Meier, $\mathrm{W}$. Bioinspired polymer vesicles and membranes for biological and medical applications. Chem. Soc. Rev. 2016, 45, 377-411.

(26) Schwarzer, T. S.; Klermund, L.; Wang, G.; Castiglione, K. Membrane functionalization of polymersomes: alleviating mass transport limitations by integrating multiple selective membrane transporters for the diffusion of chemically diverse molecules. Nanotechnology 2018, 29, 44LT01.

(27) Poschenrieder, S. T.; Schiebel, S. K.; Castiglione, K. Stability of polymersomes with focus on their use as nanoreactors. Eng. Life Sci. 2018, 18, 101-113.

(28) Poschenrieder, S. T.; Wagner, S. G.; Castiglione, K. Efficient production of uniform nanometer-sized polymer vesicles in stirredtank reactors. J. Appl. Polym. Sci. 2016, 133, 43274.

(29) Narmandakh, A.; Bearne, S. L. Purification of recombinant mandelate racemase: Improved catalytic activity. Protein Expression Purif. 2010, 69, 39-46.

(30) Nardin, C.; Hirt, T.; Leukel, J.; Meier, W. Polymerized ABA triblock copolymer vesicles. Langmuir 2000, 16, 1035-1041.

(31) Piszko, M.; Kankanamge, C. J.; Rausch, M. H.; Klein, T.; Fröba, A. P. Dynamic Light Scattering for Studying Mutual Diffusion Coefficients in Electrolyte Systems Comprised Entirely of Ions. J. Electrochem. Soc. 2020, 167, 133502.

(32) Meng, X.; Wu, J.; Liu, Z. Viscosity and Density Measurements of Diisopropyl Ether and Dibutyl Ether at Different Temperatures and Pressures. J. Chem. Eng. Data 2009, 54, 2353-2358.

(33) Poschenrieder, S. T.; Hanzlik, M.; Castiglione, K. Polymersome formation mechanism and formation rate in stirred-tank reactors. J. Appl. Polym. Sci. 2018, 135, 46077.

(34) Chênevert, R.; Pelchat, N.; Morin, P. Lipase-mediated enantioselective acylation of alcohols with functionalized vinyl esters: acyl donor tolerance and applications. Tetrahedron: Asymmetry 2009, 20, 1191-1196.

(35) Strauss, U. T.; Faber, K. Deracemization of (+/-)-mandelic acid using a lipase-mandelate racemase two-enzyme system. Tetrahedron: Asymmetry 1999, 10, 4079-4081.

(36) Melais, N.; Aribi-Zouioueche, L.; Riant, O. The effect of the migrating group structure on enantioselectivity in lipase-catalyzed kinetic resolution of 1-phenylethanol. C. R. Chim. 2016, 19, 971-977. (37) Takechi-Haraya, Y.; Sakai-Kato, K.; Goda, Y. Membrane Rigidity Determined by Atomic Force Microscopy Is a Parameter of the Permeability of Liposomal Membranes to the Hydrophilic Compound Calcein. AAPS PharmSciTech 2017, 18, 1887-1893.

(38) Kotova, E. A.; Kuzevanov, A. V.; Pashkovskaya, A. A.; Antonenko, Y. N. Selective permeabilization of lipid membranes by photodynamic action via formation of hydrophobic defects or prepores. Biochim. Biophys. Acta, Biomembr. 2011, 1808, 2252-2257. 
(39) Wang, Z.; van Oers, M. C. M.; Rutjes, F. P. J. T.; van Hest, J. C. M. Polymersome Colloidosomes for Enzyme Catalysis in a Biphasic System. Angew. Chem., Int. Ed. 2012, 51, 10746-10750.

(40) Yang, X.; Wang, Y.; Bai, R.; Ma, H.; Wang, W.; Sun, H.; Dong, Y.; Qu, F.; Tang, Q.; Guo, T.; Binks, B. P.; Meng, T. Pickering emulsion-enhanced interfacial biocatalysis: tailored alginate microparticles act as particulate emulsifier and enzyme carrier. Green Chem. 2019, 21, 2229-2233.

(41) Poschenrieder, S. T.; Schiebel, S. K.; Castiglione, K. Polymersomes for biotechnological applications: Large-scale production of nano-scale vesicles. Eng. Life Sci. 2017, 17, 58-70.

(42) Hortsch, R.; Weuster-Botz, D. Milliliter-scale stirred tank reactors for the cultivation of microorganisms. Adv. Appl. Microbiol. 2010, 73, 61-82.

(43) Riedlberger, P.; Weuster-Botz, D. New miniature stirred-tank bioreactors for parallel study of enzymatic biomass hydrolysis. Bioresour. Technol. 2012, 106, 138-146. 